\title{
Mental health in primary care
}

\author{
Focus on the Third World
}

\author{
O. O. Famuyiwa, Senior Lecturer, Department of Psychiatry, College of Medicine, \\ University of Lagos, PMB 12003, Lagos, Nigeria
}

The expression primary health care (PHC) gives the impression of an ensemble of control of communicable diseases, infant care, avoidance of insanitary personal habits and curbing environmental pollution.' These indeed constitute the main elements of the scheme but are not exclusive to it. An integral but often ignored aspect is the community dimension of mental health whose importance is underscored by the fact that a significant majority of people in the third world live in rural areas with minimal or no access to formal psychiatric facilities. In this paper, I shall review the conceptual framework and practicality of primary health care with special reference to community mental health, highlight some notable limitations to policy execution and finally propose an organisational model structure for the community mental health services within the primary health care system.

\section{Basic concepts}

The primary health care scheme has stimulated international concern for nearly a decade. Many authorities will concur with the view that the concept and practice of PHC may prove to be the longest stride in international health programming and an epoch in our quest for a viable community health service. The rudimentary processes which were seminal to the ideals of the PHC included the realisation that a substantial proportion of the population had inadequate access to health care facilities and that good health should be a right of every human being. A precursor to the PHC - the adoption of the United Nations Universal Declaration of Human Rights of the World Health Organization - after more than three decades of unaccomplished aspirations had fizzled out into virtual oblivion. The real change emerged when the fundamental tenets of the PHC were tabled at the World Health Assembly in 1977 and in September 1978 formally articulated as a series of bold proposals at an international conference in Alma Ata, USSR under the aegis of the World Health Organization. ${ }^{2}$ The provisions of the Alma Ata declaration received world-wide acclaim and were warmly welcomed by health service planners and Government functionaries endowed with social conscience and are committed to public health.

The Alma Ata declaration stipulates that firstly, people have the right and duty maximally to participate individually and in concert in the planning and execution of health services and that such services should be made accessible to every section of the community. Secondly, high premium should be attached to the individual's self-reliance in the initiation and sustenance of the PHC with utilisation of national and local resources and health education targeted to populations at risk. The corollary of these objectives is that through guided community participation, measures can be taken to facilitate promotive, preventive, curative and rehabilitative health matters. A notable misconception is to regard the PHC as an alternative to national health service.

The Alma Ata paper reaffirms a 1975 resolution of the World Health Assembly when it states that the PHC is the point of entry for the individual to the national health service and not the point of first contact with any particular health worker, and as such it should be regarded as an integral component of the national health service. One of the paramount facets of the PHC concept, which unfortunately is easily overlooked, is that it draws awareness to the need to expand the traditional narrow conception-which views health care delivery system in terms of the activities of doctors, nurses, social workers and allied medical personnel, drug prescribing and prophylactic measure such as immunisation - to include with a sense of purpose, effort on the part of national governments to increase the income per capita through constructive economic measures with a silhouette of national consciousness, adequate production and distribution of food, provision of pipeborne water and consistent environmental sanitation. Since its endorsement by the World Health Assembly in May 1979, the Alma Ata declaration has enjoyed considerable credibility in the USSR, shown evidence of its workability and become a stimulus for other countries, particularly developing ones.

A number of African countries have progressed beyond the conceptual level to initial execution of the scheme. In Ghana, there had been a call for stemming 
health education and intensively preparing the community. ${ }^{3}$ Botswana, Lesotho and Zambia have begun to train hospital nurses in basic community psychiatric nursing and to utilise local traditional values for outreach community mental services. ${ }^{4}$ In Nigeria and Gambia the Government had given impetus to public awareness on PHC. In the College of Medicine of the University of Lagos, a whole academic session (about a fifth of the undergraduate medical curriculum) is devoted to teaching, demonstration and field work in PHC. One important move within Nigeria was the scientific approach to PHC adopted by two workers ${ }^{5}$ in which baseline social and demographic data were obtained, by a survey of a typical rural population in the South West area. This exercise is justified by the importance of epidemiology in planning health service and identifying high-risk groups. For instance, in the survey female illiteracy was estimated to be $78 \%$ and plans are now being made to 'develop a functional literacy programme with a health focus'. As a comprehensive PHC includes community mental health it is instructive to define some of its important features as ordinarily conceptualised.

\section{Community mental health}

Few people realise that community mental health had been in existence in Africa before the advent of western medicine. Some aspects which come to mind include folk therapy, the sharing of emotions among members of syncretic churches, the prophetic value of oracle in the amelioration of the effect of, and increased resilience to, stressful events, the multiple parent figures which provide alternative substitute care in traumatic childhood and the primary prevention attitude of opposition to choice as marital partner of an individual with personal or family history of mental illness! These cultural processes unfortunately do not lend themselves to objective evaluation. Organised concern with the need for a properly structured and articulated community mental health service in Africa was stimulated by the fortitude of the first generation African psychiatrists and the success of the Aro Village system. This system consists of a group of villages where patients share with their close relatives a relatively mundane life situation and have access to psychiatric treatment facilities. There is evidence that illness-outcome in functional psychoses was better in patients treated in the village system than in a psychiatric department of a general hospital. ${ }^{6}$

In more recent times, in most countries, particularly of the third world, formal psychiatric education and health service planning have acknowledged the utility in, and impetus for, the promotion of public mental hygiene by well-programmed community psychiatric services but, in pragmatic terms, such services where they exist at all appear to be poorly organised and inadequately staffed and funded. Research in community psychiatry. lags behind research in biological and psychosocial themes, yet the meaningful application of findings from the scientific enquiries into these themes cannot be divorced from committed attention to community based mental health programmes. Community psychiatry became a recognised discrete subspecialty with the passage in the US of the Community Mental Health Centres Act of 1963, and by the mid 1970s, nearly $\mathbf{4 0 0}$ such centres were functioning all over the $\mathrm{USA}^{7}$ the Act being a culmination to at least three historical developments. Firstly, the Geel community in Belgium, established in 1950 initially as a religious institution, made provisions for partial hospital admittance for patients and their families. Secondly, Adolf Meyer in 1913 argued a strong case for the establishment of a community-based health centre which was to embrace agencies (such as the police, social welfare department, and schools) affiliated to a psychiatic hospital to form a single health district. Thirdly, the Mental Health Treatment Act of 1930 in Britain directed local government authorities to make positive changes regarding outpatient's follow-up of discharged patients and the World Health Organization in 1953 recommended that adequate psychiatric staff be provided in hospitals to enable psychiatrists to attend to community services. $^{7}$

Some would feel that it is illusory at the present time to talk of community psychiatry in most developing countries where the basic conventional mural psychiatric service structure and organisation are yet to be satisfactorily developed. However, hospital psychiatric practice on the one hand and community psychiatric service on the other hand are closely intertwined with reciprocal values and deficiences.

At this juncture, it should be instructive to enumerate the major characteristics of a community mental health service which are readily applicable to diverse socio-cultural settings and this will be done with brief discursive notes on their workability in the third world setting.

\section{(a) Service population}

This is an idea obtained from public health diction. The USA federal programme suggested a geographical area of not less than 25,000 people and no more

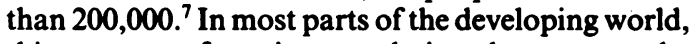
this concept of service population does not at the present time hold true, mainly because the available psychiatric facilities are not evenly distributed and mobility of service recipients is no respecter of political or administrative boundaries. Furthermore, accurate demographic data are hard to come by. One way to minimise these limitations is to establish small community units in each state or province with each 
unit affiliated to the psychiatric division of the nearest general hospital. Such psychiatric divisions have not been found to be administratively difficult to set up and the issue of personnel can be tackled in the short-term by scheduling the nearest psychiatrist to consult periodically at such divisions. Experiences in the Western Nigeria Health Services in the 1960s demonstrated the feasibility and wisdom of this approach.

\section{(b) Service scope}

This should be comprehensive and include inpatients, out-patients, crisis intervention, partial hospitalisation and educational facilities. Such a scope of activities is certainly not realisable in most developing countries at present because of economic constraints and relative insensitivity of some governments to the needs of good psychiatric services. Perhaps the utilisation of non-psychiatric centres and paraprofessionals needs to be favourably considered. Furthermore, constructive pressure from psychiatrists and other mental health professionals should be applied for the formulation of legislations to facilitate the material and moral backing to any well-prepared community mental health service programme.

\section{(c) Service continuity}

Following successful acute treatment, the patient's care must continue extra-murally. Successful continuity depends on sustaining commitment to the patient by the caring team, and smooth and effective liaison with other available non-mental health services. Since most developing countries do not have organised community mental health service as such, continuity of care can be fostered through the support of general practitioners, special educational agencies and voluntary organisations. Additional personnel could be social welfare officers who perform routine administrative rather than professional duties in ministries of social welfare. It should be useful to implore Government to deploy some of these social welfare workers to the community mental health field at least on a part-time basis in the first instance. It cannot be over-emphasised that service scope and service continuity cannot be readily achieved in most developing countries in view of preexisting economic difficulties, misguided priorities in national planning and unpredictability of political administration. However, some advances can be acomplished by innovatively rescheduling the responsibilities of available health manpower towards preventive rather than curative goals.

\section{(d) Prevention}

This has three inter-related dimensions. Primary prevention is the most fundamental and entails iden- tifying and eliminating factors which can cause mental illness; implying that measures could be taken to forestall the onset of mental illness. The whole concept emphasises the promotion of mental health and the protection against occurrence of specific dysfunctional psychological states. Two major handicaps are the low agreement on what is mental health and its differentiation form mental illness. The blurred boundary remains one of the most practical problems in mental health care delivery. However, we can stick to a general agreement that mental health is not simply the absence of mental illness but as Jahoda puts it, "a relatively constant and enduring function of personality". Secondary prevention is directed to the early diagnosis and prompt treatment which embodies screening large populations for illness cases, developing crisis intervention programmes, educating the general population with the object of raising the level of awareness about signs of mental illness and prompt institution of appropriate treatment. Tertiary prevention is the prevention of long-term disability arising from mental illness. This applies to chronically disabled mental patients who often present with a variety of clinical problems such as mutism, abnormal posturing, indecent behaviour, unexplainable aggression, and poor integration into society. The severely deteriorated stage should not be reached because of its potential irreversibility. In contrast to the primary and secondary prevention, tertiary prevention is more readily achievable.

\section{(e) Research/evaluation}

This important characteristic refers to periodic exercises whereby the success or failure of any community mental health programme is assessed and necessary changes effected. With effective and well-validated evaluation approaches, limitations, deficiencies and assets are identifiable such that new stratagems can be formulated.

\section{(f) Service-recipient participation}

A major consequence of this characteristic is enshrined in the PHC as mentioned above. It should be accepted that Primary Health Care is an integrated service and the incorporation of mental health into it should not be different from that of other specialities of medicine, except that the abstract nature and the causation of mental diseases and pre-existing societal attitudes call for some changes such as in personnel training and the modalities of mental health education.

\section{Organisational model}

In the relatively short history of community mental health, two basic forms of organisational modes have 
emerged. These models are flexible such that either can be applied to different health care service systems. Firstly, there is the model in which several community mental centres are built principally from private establishment source. Secondly, we have a model whereby a community mental health centre is created within a general hospital. The second model seems to be economical and fits better with the economic situation and cultural system in the third world countries. Its main advantage is that the maintenance of the centre would be undertaken by, and necessary administrative support obtained within, the provisions of the hospital. Such a corporate institution would also cater for the medical and surgical needs of psychiatric patients. Also with implementation of this model, the stigma attached to mental health is minimised by the 'protective' effect of the general hospital structure. A major limitation of the model,

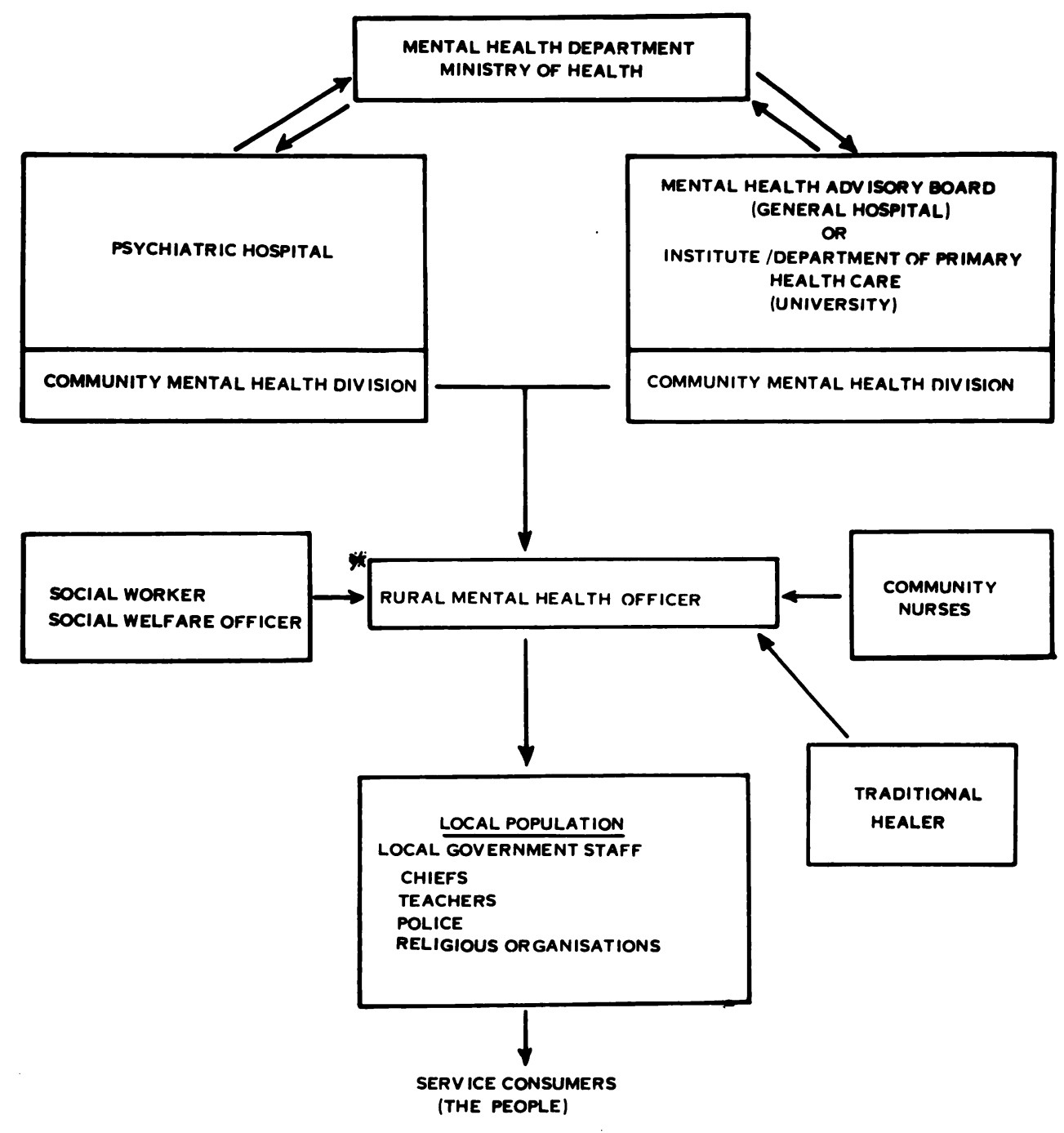

PROPOSED SCHEME OF COMMUNITY HEALTH SERVICE WITHIN THE FRAMEWORK OF THE PRIMARY HEALTH CARE (PHC).

- equivalent of VILLAge heALTH wORKER IN THE PHC.

FIG. 1. Proposed scheme of community health service within the framework of the primary health care (PHC). 
however, is the probable insinuation of the civil service bureaucracy which generally causes tardiness in policy execution and some degree of censorship to set aspirations and goals. One way to minimise this problem is the setting up of a mental health advisory committee which would guide the hospital management in its commitment to the community mental health division. Existing mental hospitals can have more than one community mental health unit subserving extensive catchment areas and also organise programmes geared primarily to training community mental health personnel. The dearth and poor distribution of psychiatric and medical personnel in Africa means this model is workable only in very few districts of each country but for any innovative idea to germinate there must be a beginning.

Regarding the modalities of the mental health programme of the PHC, specific approaches would vary from one developing country to another. However, there should be some basic principles which should prove helpful. A pragmatic approach could be that the staff of the community mental health units, with the mental health officer and with support of the local chiefs and other community leaders, organise educative sessions for local populations on preventive themes such as the importance of parental care and maternal health to the development of the child, the dangers of drug abuse, early recognition of mental dysfunctional states, correction of negative attitudes to, and misconception about, mental disorders, and also provide guides as to utilisation of available curative facilities. The initial target population should be special groups like the school teachers, who would be required to impart their acquired mental health knowledge to other community workers, and the latter in turn would educate other community groups like the parent-teachers' association, voluntary organisations and religious bodies, thereby building an education pyramid. The focal person is the rural mental health officer. He is a person with basic educational qualifications, like the successful completion of at least secondary education, who received a short course, of say one year, in basic mental health, including counselling. A career ladder should be created for the mental health officers and should be tailored on the pattern which obtains for public service in each country. He is to receive support from existing community nurses and social welfare officers (where they are available). Dialogues between the traditional healers and orthodox medical personnel, particularly at the level of the community mental health officer, should be encouraged, the idea being to extract those traditional healing approaches of proven value and marry them with orthodox therapeutic measures as has been practised in China and India. The scheme being proposed (see Fig. 1) will have the mental health division of the ministry of health as the main co-ordinator and the 'middleman' between the mental health component of the PHC and the central government.

Well-programmed primary health care in Africa with particular reference to mental health has a significantly increased chance of success in that there is good evidence that the prognosis of functional mental disorders is better in the developing countries that the industrialised west. ${ }^{8}$ It can, therefore, be postulated that the socio-cultural matrix of the third world contains some factors which augur for the favourable course of mental illness. These factors are yet to be precisely identified but it is generally believed that the mores and the kinship extended family system with its support quality are the most probable therapeutic ingredients.

Finally, national governments must demonstrate commitment to the PHC in national planning and should realise that the PHC is not in the exclusive domain of the health sector but requires interministerial fraternisation. It is indeed operationally linked with social and economic growth and development, and, if properly managed, should contribute in Africa to improvement in the physical Quality of Life index - an aggregate measure encompassing life expectancy at age one year, infant mortality and literacy. One hopes that post-independence political instability in black Africa and rapid changes of administration, with consequent non-completion of projects and programmes and reversal of policies, do not recur to constitute a hindrance to achieving the lofty goals of primary health care.

\section{References}

${ }^{1}$ Gullbert, J. J. (1981) Educational Handbook for Health Personnel. Geneva: World Health Organization (Offset publication No. 35).

${ }^{2}$ Primary Health Care: $A$ report of the International Conference on Primary Health Care, Alma Ata, USSR. 6-12 September, 1978. Geneva: World Health Organization, 1978.

${ }^{3}$ PAPPOE, M. E. (1987) Primary health care: a challenge to health education in Ghana. Ghana Medical Journal, September, 29-36.

${ }^{4}$ WANKIIRI, V. B. (1984) Mental health and psychiatric nursing in Africa. World Health Forum, 5, 334-337.

${ }^{5}$ BAMISAYYE, A. \& JoHNSON, T. O. (1988) Planning PHC for community: a baseline survey provides essential data. Tropical Doctor, January, 36 \& 37.

${ }^{6}$ Lambo, T. \& Dastoor, D. P. (1972) Evaluation of the community psychiatry experiment at Aro Village, Abeokuta, Nigeria. Mimeograph, Behaviour Sciences Research Unit. University of Ibadan.

'WESTON, D. W. (1975) Development of community psychiatry concepts. Comprehensive Textbook of Psychiatry, 2, 2310-2323.

${ }^{8}$ WHO Report (1978) The International Pilot Study of Schizophrenia. Vol. 1, Geneva: World Health Organization. 